\title{
Duality and randomization in nonlinear programming
}

\author{
Jean B. Lasserre*
}

(Received 4 May 2000, revised 2 Nov 2000)

\section{Abstract}

We consider the NLP optimization problem

$$
\mathrm{P} \mapsto \inf _{x}\left\{f_{0}(x) \mid f_{i}(x) \leq b_{i}, i=1, \ldots, m\right\}
$$

and discuss the duality gap between $\mathrm{P}$ and

$$
\mathrm{D} \mapsto \sup _{\lambda \geq 0} \inf _{x}\left\{f_{0}(x)+\sum_{i=1}^{m} \lambda_{i}\left[f_{i}(x)-b_{i}\right]\right\} .
$$

*LAAS-CNRS, 7 Avenue du Colonel Roche, 31077 Toulouse Cédex, France. mailto:lasserre@laas.fr

${ }^{0}$ See http: //anziamj . austms.org.au/V42/E017 for this article and ancillary services, (C) Austral. Mathematical Soc. 2001. Published 21 January 2001. 
The convex problem $\mathrm{D}$ is in fact the dual of a "relaxed" version of $\mathrm{P}$ via "randomization" which permits to give a simple interpretation for the presence or absence of a duality gap in the general case. Several particular cases are also discussed and the case of homogeneous functions is given special attention.

\section{Contents}

1 Introduction

2 On the duality gap $\quad$ E32

2.1 The dual $D \ldots \ldots \ldots$. . . . . . . . . . . . . E33

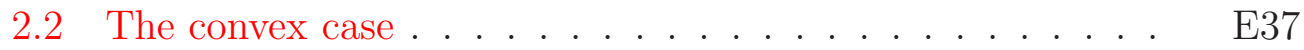

2.3 On the duality gap ................ . . . E40

2.4 The (general) quadratic case ............ E44

3 The homogeneous case $\quad$ E47

3.1 Example: Pure quadratic optimization . . . . . . . . E50

References

E53

A Proof of Theorem 1

$\begin{array}{lr}\text { B Proof of Theorem 2 } & \text { E59 }\end{array}$ 
C Proof of Theorem 3

E62

D Proof of Theorem 6

E65

\section{Introduction}

Consider the standard Nonlinear Programming Problem (NLP)

$$
\mathrm{P} \mapsto \inf _{x}\left\{f_{0}(x) \mid f_{i}(x) \leq b_{i}, i=1,2, \ldots, m\right\}
$$

where $f_{i}: R^{n} \rightarrow R, i=0,1, \ldots, m$, are all continuous functions. It is well-known that

$$
\sup \mathrm{D} \leq \inf \mathrm{P}=\inf \mathrm{P}^{*}
$$

where

$$
\mathrm{P}^{*} \mapsto \inf _{x} \sup _{\lambda \geq 0}\left\{f_{0}(x)+\sum_{i=1}^{m} \lambda_{i}\left[f_{i}(x)-b_{i}\right]\right\}
$$

and

$$
\mathrm{D} \mapsto \sup _{\lambda \geq 0} \inf _{x}\left\{f_{0}(x)+\sum_{i=1}^{m} \lambda_{i}\left[f_{i}(x)-b_{i}\right]\right\}
$$


When permitted, the interchange of "inf" and "sup" operators (that is, the equivalence of (2) and (3)) characterizes the absence of a duality gap and optimal solutions (if any) are saddle points of the Lagrangian (see e.g. [8], [10]). Except for the convex case, i.e. when all the $f_{i}$ are convex (and under a constraint qualification), there is no systematic approach to decide whether or not there is a duality gap. Hence, identifying instances for which the absence of a duality gap is guaranteed is a challenging problem. This is not surprising for $\mathrm{P}$ and $\mathrm{D}$ are very different in nature. Solving $\mathrm{P}$ is in general a difficult global optimization problem whereas D is a convex problem, easier to solve in principle. In particular cases, some authors have obtained interesting results (see e.g. quadratic and pure quadratic problems in [2], [9], [13] and the references therein).

The goal of this paper is to provide some insights on this duality gap issue. We show that both $\mathrm{D}$ and $\mathrm{P}^{*}$ are in fact derived from "relaxed" versions of P. D is obtained as the dual of a linear "randomized" version PP of $\mathrm{P}$, the analogue of the familiar "relaxed control" procedure in control (see e.g. [3], [11], [15]), that yields the concept of "generalized solutions" (or "Young measures") to the original problem, whereas $\mathrm{P}^{*}$ is obtained by embedding $\mathrm{P}$ into a larger (but equivalent) problem. In turn, PP is also the dual of $\mathrm{D}$ and under weak conditions, there is no duality gap between $\mathrm{D}$ and PP. In particular, it is shown that to every optimal solution $\lambda$ of the dual problem D, corresponds an optimal "randomized" solution $\mu$ to the primal problem PP ( $\mu$ may be interpreted as a "generalized" solution to P). In this approach, the absence of a duality gap between $\mathrm{P}$ and $\mathrm{D}$ in the convex case under Slater's constraint qualification appears as an immediate consequence 
of Jensen's inequality.

In a sense, PP is a "regularized" (or "convex") version of P, pretty much like $f^{* *}$ is a regularized version of a nonconvex function $f$ with LegendreFenchel transformed $f^{*}$. This "randomization" (or "convexification") procedure has been successfully applied to some problems in Control Theory, Economics (see e.g. [11], [15]). Here, we emphasize the duality point of view. More precisely, it is shown that under weak conditions, there is no duality gap between PP and D. In addition, when both D and PP are solvable, there is a probability distribution $\mu$ on the the set $X(\lambda)$ of global minima of the Lagrangian $L(., \lambda)$ (with $\lambda$ any optimal solution of D), such that $\int f_{i} d \mu \leq b_{i}$ $i=1,2, \ldots, m$ and $\int f_{0} d \mu=\max \mathrm{D}$. In other words, $\mu$ is an optimal solution of a "relaxed" version of P, i.e., when the constraints and the objective are "averaged out" with respect to a probability measure. In general, this "averaging procedure" yields generalized solutions with a strictly better cost than usual solutions. An even finer characterization of optimal generalized solutions is obtained via Caratheodory Theorem when the set $X(\lambda)$ is compact.

As a Corollary, there is no duality gap between $\mathrm{D}$ and $\mathrm{P}$ if and only if all the global minimizers of $\mathrm{P}$ belong to $X(\lambda)$ and checking the absence of a duality gap reduces to check whether there is a feasible solution of $\mathrm{P}$ in $X(\lambda)$ such that a complementary condition holds. For instance, when the $f_{i}$ 's are all quadratic, first solve D, obtain $\lambda$ and then check whether there is a feasible solution $x^{*}$ of $\mathrm{P}$ that solves a quadratic system of equations/inequations in a space of smaller dimension (cf. Corollary 4 of Section 2). This is in contrast 
to the trial and error method which consists in first finding a Karush-KuhnTucker point $\left(x^{*}, \lambda^{*}\right)$ of $\mathrm{P}$ and checking afterwards whether $x^{*}$ is also a global minimizer of the Lagrangian $L\left(., \lambda^{*}\right)$.

Some other particular cases are also investigated. For instance, if $X(\lambda)$ is a singleton then solving the nonconvex problem $\mathrm{P}$ reduces to solving the convex problem D. The general quadratic case is also investigated. The dual $\mathrm{D}$ is an LMI problem whose dual $\mathrm{D}^{*}$ is a well-known relaxation of $\mathrm{P}$ (see Boyd and Vandenberghe [6]). The optimal values of PP and $\mathrm{D}^{*}$ coincide and optimal solutions of PP with finite support provide a natural interpretation of the optimal solutions of $\mathrm{D}^{*}$ in terms of "randomization".

Finally, the case of homogeneous functions is considered. The dual D takes a particular form and the solvability of $\mathrm{PP}$ is obtained under weak conditions. In addition, when $X(\lambda)$ is a one-dimensional cone, then $\min \mathrm{P}=$ max D and solving $\mathrm{P}$ reduces to solving a convex problem (an LMI problem in case all the $f_{i}$ 's are quadratic forms).

\section{On the duality gap}

Consider the following optimization problem in $X:=R^{n}$ :

$$
P \mapsto f^{*}:=\inf \left\{f_{0}(x) \mid f_{i}(x) \leq b_{i}, \quad i=1,2, \ldots, m\right\}
$$


where $f_{i}: X \rightarrow R, i=0,1, \ldots, m$ are all continuous functions. Denote by $S$ the feasible set of P, i.e.,

$$
S:=\left\{x \in X \mid f_{i}(x) \leq b_{i} \quad i=1,2, \ldots, m\right\} .
$$

Assumption A: $\forall i=1,2, \ldots, m$, there exists $x_{i} \in S$ such that $f_{i}\left(x_{i}\right)<b_{i}$.

Note that Assumption A is just Slater's constraint qualification whenever the functions $f_{i}$ are convex $i=1,2, \ldots, m$.

\subsection{The dual $D$}

Consider now the following "relaxation" PP of P:

$$
\operatorname{PP}\left\{\begin{aligned}
\inf _{\mu \in \mathcal{M}(X), \mu \geq 0} \int f d \mu & \\
\int\left[f_{i}(x)-b_{i}\right] \mu(d x) & \leq 0, \quad i=1,2, \ldots, m, \\
\mu(X) & =1
\end{aligned}\right.
$$

where $\mathcal{M}(X)$ is the Banach space of signed Borel measures on $\mathcal{B}$ (the Borel $\sigma$-field on $X$ ), equipped with the total variation norm.

Obviously, $\mathrm{PP}$ is a relaxation of $\mathrm{P}$ via randomization since for every admissible point $x \in S$, the Dirac measure $\mu:=\delta_{x}$ concentrated at the point $x$, 
is admissible in $\mathrm{PP}$ with corresponding value $f(x)$ and therefore, inf $\mathrm{PP} \leq f^{*}$. In fact, this relaxation is a "convexification" of $\mathrm{P}$, the analogue of the "relaxed controls" procedure in control (see e.g. [3], [11], [15] and the references therein). The advantage of doing this is that the linear problem PP admits a natural "dual" linear problem D introduced below:

$$
\mathrm{D} \mapsto \sup _{\lambda \geq 0, \gamma}\left\{\gamma \mid f_{0}(x)+\sum_{i=1}^{m} \lambda_{i}\left[f_{i}(x)-b_{i}\right] \geq \gamma, \quad \forall x \in X\right\},
$$

or equivalently

$$
\mathrm{D} \mapsto \sup _{\lambda \geq 0} \inf _{x}\left\{f_{0}(x)+\sum_{i=1}^{m} \lambda_{i}\left[f_{i}(x)-b_{i}\right]\right\}
$$

which the usual "dual" considered in NLP. In fact D and PP are dual of each other.

More precisely, with $w:=1+\max _{i=1, \ldots, m}\left|f_{i}\right|$, and following Anderson and Nash [1], let $(\mathcal{X}, \mathcal{Y})$ and $(\mathcal{Z}, \mathcal{W})$ be two dual pairs of vector spaces where

$$
\mathcal{X}:=\left\{\mu \in \mathcal{M}(X)\left|\int w d\right| \mu \mid<\infty, i=0,1, \ldots, m\right\},
$$

(where $|\mu|$ denotes the total variation of $\mu$ ), and

$$
\mathcal{Y}:=\left\{f: R^{n} \rightarrow R \mid \sup _{x \in R^{n}} \frac{|f(x)|}{w(x)}<\infty\right\}
$$


whereas, $\mathcal{W}=\mathcal{Z}:=R^{m}$. The pair $(\mathcal{X}, \mathcal{Y})$ is in duality via

$$
\langle\mu, h\rangle=\int h d \mu, \quad \mu \in \mathcal{X}, h \in \mathcal{Y} .
$$

Introducing the linear maps

$$
\begin{aligned}
T: \mathcal{X} & \longrightarrow \mathcal{Z} \\
\mathcal{Y} & \longleftarrow \mathcal{W}: \widehat{T}
\end{aligned}
$$

where

$$
\mu \mapsto T \mu:=\left[\begin{array}{c}
\int\left(f_{1}-b_{1}\right) d \mu \\
\vdots \\
\int\left(f_{m}-b_{m}\right) d \mu
\end{array}\right] \quad \mu \in \mathcal{X}
$$

and

$$
\lambda \mapsto \widehat{T} \lambda:=\sum_{i=1}^{m} \lambda_{i}\left[f_{i}-b_{i}\right] \quad \lambda \in \mathcal{Z},
$$

the linear program PP reads

$$
\mathrm{PP} \mapsto \inf _{\mu \in \mathcal{X}, \mu \geq 0}\left\{\left\langle f_{0}, \mu\right\rangle \mid T \mu \leq 0 ;\langle 1, \mu\rangle=1\right\},
$$

whereas the linear program $\mathrm{D}$ reads

$$
\mathrm{D} \mapsto \sup _{(\lambda, \gamma) \in \mathcal{Z} \times R, \lambda \geq 0}\left\{\gamma \mid \gamma-\widehat{T}(\lambda) \leq f_{0}\right\}
$$


which is just (7). It is straightforward to check that $R+\widehat{T}(\mathcal{Z}) \subseteq \mathcal{Y}$ so that the linear map $T_{1}: \mathcal{X} \rightarrow \mathcal{Z} \times R$ defined by $\mu \mapsto T_{1} \mu:=(T \mu,\langle 1, \mu\rangle)$ is continuous for the respective weak topologies $\sigma(\mathcal{X}, \mathcal{Y})$ and $\sigma(\mathcal{Z} \times R, \mathcal{W} \times R)$ (see e.g. [7]).

On the other hand, consider now the other following equivalent version $\mathrm{P}^{*}$ of $\mathrm{P}$,

$$
\mathrm{P}^{*} \mapsto \inf _{u \geq 0, x}\left\{u f_{0}(x) \mid u\left[f_{i}(x)-b_{i}\right] \leq 0, i=1,2, \ldots, m ; u=1\right\} .
$$

Equivalently,

$$
\mathrm{P}^{*} \mapsto \inf _{x} \inf _{u \geq 0}\left\{u f_{0}(x) \mid u\left[f_{i}(x)-b_{i}\right] \leq 0, i=1,2, \ldots, m ; u=1\right\},
$$

and noting from LP duality that the dual of the LP problem

$$
\inf _{u \geq 0}\left\{u f_{0}(x) \mid u\left[f_{i}(x)-b_{i}\right] \leq 0, i=1,2, \ldots, m ; u=1\right\},
$$

is just

$$
\sup _{\lambda \geq 0, \gamma}\left\{\gamma \mid f_{0}(x)+\sum_{i=1}^{m} \lambda_{i}\left[f_{i}(x)-b_{i}\right] \geq \gamma\right\},
$$

one obtains the the following equivalent formulation

$$
\mathrm{P}^{*} \mapsto \inf _{x} \sup _{\lambda \geq 0}\left\{f_{0}(x)+\sum_{i=1}^{m} \lambda_{i}\left[f_{i}(x)-b_{i}\right]\right\},
$$

which is (2). We now introduce the following condition: 
Condition B: There exist nonnegative coefficients $\lambda_{i}, i=1,2, \ldots, m$, such that

$$
\inf _{x \in X}\left\{f_{0}(x)+\sum_{i=1}^{m} \lambda_{i} f_{i}(x)\right\}>-\infty .
$$

We first consider the solvability of $\mathrm{D}$ and the absence of a duality gap between D and PP, under Assumption A and condition B.

Theorem 1 Let Assumption $A$ and Condition B hold, then

$$
\sup D=\max D=\inf P P .
$$

The proof is relegated to Appendix A. Of course, the main issue of interest is to determine when inf $\mathrm{PP}=\inf \mathrm{P}=f^{*}$ holds or to gain some insight into the presence of a duality gap, i.e. when inf $\mathrm{PP}<\inf \mathrm{P}$.

For convex problems recall that under Slater's condition, inf PP $=$ inf $\mathrm{P}$ (see e.g. [10]). However, we provide below a simple proof of this result that simply uses Jensen's inequality.

\subsection{The convex case}

In this section we assume that $f_{i}$ are convex $i=0,1, \ldots, m$. Assumption A becomes Slater's constraint qualification under which max D = inf P, a wellknown result. 
(i) The fact that inf PP $=$ inf $\mathrm{P}$ is in fact an immediate consequence of Jensen's inequality. In case $X$ would be a compact convex subset of $R^{n}$, then $\int x d \mu=: E_{\mu}(x) \in X$ is well defined and Jensen's inequality applies, that is,

$$
\int f d \mu \geq f\left(E_{\mu}(x)\right)
$$

for every continuous convex function $f$ (see e.g. Perlman [12]). Therefore, to every admissible solution $\mu$ of PP, one may associate an admissible solution $E_{\mu}(x)$ of $P$ with a lower cost.

When $X:=R^{n}$, the proof also uses a compactness argument. For the problems $P P_{i}$ defined in the proof of Theorem 1, $\mu_{i}$ defines a probability measure on $K_{i}$ with corresponding expectation operator $E_{\mu_{i}}$. As $K_{i}$ is compact and can be chosen convex, the random vector $x \in K_{i}$ is $\mu$-integrable, and since $K_{i}$ is convex, Jensen's inequality is valid and yields

$$
\int f_{k} d \mu_{i} \geq f_{k}\left(E_{\mu_{i}}(x)\right), \quad k=0,1, \ldots, m .
$$

In particular,

$$
0 \geq \int\left(f_{k}(x)-b_{k}\right) \mu_{i}(d x) \geq f_{k}\left(E_{\mu_{i}}(x)\right)-b_{k}, \quad k=1,2, \ldots, m,
$$

so that the point $y:=E_{\mu_{i}}(x)$ is feasible in $\mathrm{P}$ with value $f_{0}(y) \leq \gamma_{i}$. Therefore, inf $\mathrm{P}=\inf \mathrm{PP}_{i}$ for all $i$. As inf $\mathrm{PP}_{i} \rightarrow \inf \mathrm{PP}=\max \mathrm{D}$ we obtain inf $\mathrm{P}=\max \mathrm{D}$. 
(ii) The fact that there is no duality gap between PP and D also follows from Slater's constraint qualification which is an "interior point" condition for absence of a duality gap (for instance, invoke Theorem 3.13, p. 55 in Anderson and Nash [1]).

(iii) Assume that $x^{*}$ is an optimal solution of P. With $\lambda^{*}$ an optimal solution of $\mathrm{D}$ we obtain,

$$
f_{0}\left(x^{*}\right)+\sum_{i=1}^{m} \lambda_{i}^{*}\left[f_{i}\left(x^{*}\right)-b_{i}\right] \geq \max \mathrm{D}=f_{0}\left(x^{*}\right),
$$

which yields

$$
\sum_{i=1}^{m} \lambda_{i}^{*}\left[f_{i}\left(x^{*}\right)-b_{i}\right]=0,
$$

since $f_{i}\left(x^{*}\right) \leq b_{i}$ for all $i$. Hence, $x^{*}$ is a saddle point of the Lagrangian $L\left(., \lambda^{*}\right)$. In addition, if the $f_{i}$ are all differentiable,

$$
\nabla f_{0}\left(x^{*}\right)+\sum_{i=1}^{m} \lambda_{i}^{*} \nabla f_{i}\left(x^{*}\right)=0
$$

so that $\left(x^{*}, \lambda^{*}\right)$ is a Karush-Kuhn-Tucker (KKT) point of $\mathrm{P}$.

In the general (non convex) case, $\sup \mathrm{D} \leq \inf \mathrm{P}$. The case where max $\mathrm{D}=$ min PP is of particular interest for we are then able to provide some further insight on a possible duality gap between $\mathrm{P}$ and $\mathrm{D}$. It first requires the solvability of PP. 
Theorem 2 Let Assumption A and Condition B hold. In addition, assume that

(a) $f_{m}(x)$ is inf-compact, that is, the levels sets $\left\{x \mid f_{m}(x) \leq r\right\}$ are compact for every $r \in R$;

(b) $\left|f_{i}(x)\right| /\left(M+f_{m}(x)\right) \rightarrow 0$ as $|x| \rightarrow \infty$, for all $i=0,1, \ldots, m-1$, and some $M>0$.

Then $P P$ is solvable and $\max D=\min P P$.

The proof is relegated to Appendix B. The index $m$ in Conditions (a)(b) in Theorem 2 is arbitrary and can be any in the set $\{0, \ldots, m\}$. These conditions (a)-(b) are particular cases of the property $(\gamma)$ stated in Balder [3] in a more general context, that is, (a)-(b) imply that $f_{i}, i=0,1, \ldots, m-1$ have the property $(\gamma)$ with respect to $f_{m}$.

Observe that the conditions (a)-(b) rule out the cases where all the $f_{i}$ are of same nature (e.g. linear, quadratic, ...). In this case, ad hoc conditions must be found to ensure solvability of PP.

\subsection{On the duality gap}

We now provide, proved in Appendix C, several characterizations of the optimal solutions of the relaxed version $\mathrm{PP}$ of $\mathrm{P}$, when they exist. This will 
help understand the presence of a duality gap between $\mathrm{P}$ and $\mathrm{D}$.

Theorem 3 Let Assumption A hold and Condition $B$ be true, then

(a) for every solution $\mu^{*}$ of PP and every solution $\lambda^{*}$ of D, one has:

$$
f_{0}(x)+\sum_{i=1}^{m} \lambda_{i}^{*} f_{i}(x)=\min _{y \in X}\left\{f_{0}+\sum_{i=1}^{m} \lambda_{i}^{*} f_{i}\right\} \quad \mu^{*} \text { a.e. }
$$

and

$$
\lambda_{i}^{*}\left[\int f_{i} d \mu^{*}-b_{i}\right]=0, \quad i=1,2, \ldots, m .
$$

In addition, if the $f_{i}$ are all differentiable, then

$$
\nabla f_{0}(x)+\sum_{i=1}^{m} \lambda_{i}^{*} \nabla f_{i}(x)=0, \quad \mu^{*} \text { a.e. }
$$

Hence, $\mu^{*}$ is concentrated on the global minimizers of the Lagrangian $L\left(., \lambda^{*}\right)$.

(b) If the set of global minimizers $X\left(\lambda^{*}\right)$ of the Lagrangian $L\left(., \lambda^{*}\right)$ is compact, then every optimal solution $\mu^{*}$ of PP is a convex combination of at most $s+1$ Dirac measures $\delta_{x_{k}}, x_{k} \in X\left(\lambda^{*}\right), k=1, \ldots, s+1$, where $s$ is the number of active constraints in PP at $\mu^{*}$, and

$$
\nabla f_{0}\left(x_{k}\right)+\sum_{i=1}^{m} \lambda_{i}^{*} \nabla f_{i}\left(x_{k}\right)=0 \quad k=1, \ldots, s+1 .
$$


When PP is solvable, Theorem 3 provides some insight into the absence of a duality gap when $\mathrm{P}$ is solvable, i.e. when $\max \mathrm{D}<\min \mathrm{P}=f_{0}\left(x^{*}\right)$ where $x^{*}$ is a global minimizer of $\mathrm{P}$. In this case, there is a probability distribution $\mu^{*}$ on the set of global minimizers of the Lagrangian $L\left(., \lambda^{*}\right)$ such that "averaging out" with respect to $\mu^{*}$ yields a better result, i.e., the average values $\int f_{i} d \mu^{*}$ satisfy

$$
\int f_{0} d \mu^{*}<f\left(x^{*}\right) ; \quad \int f_{i} d \mu^{*} \leq b_{i}, \quad i=1,2, \ldots, m .
$$

Note in parenthesis that if $\max \mathrm{D}<\min \mathrm{P}$ and $\left(x^{*}, \lambda\right)$ is a KKT point of $\mathrm{P}$ with $x^{*}$ a global minimizer, then $x^{*}$ is not a global minimizer of the Lagrangian $L(., \lambda)$. Indeed, otherwise with $\gamma:=f_{0}\left(x^{*}\right)$, the point $(\lambda, \gamma)$ would be feasible for $\mathrm{D}$, so that $\max \mathrm{D}=\min \mathrm{P}$, a contradiction. One retrieves the well-known result that if $x^{*}$ and $\lambda^{*}$ are optimal solutions of $\mathrm{P}$ and $\mathrm{D}$ respectively, then there is no duality gap if and only if $\left(x^{*}, \lambda^{*}\right)$ is a saddle point of the Lagrangian.

The following result characterizes the absence of a duality gap.

Corollary 4 Let Assumption $A$ and Condition $B$ hold. Let $\left(\lambda^{*}, \gamma^{*}\right)$ be an optimal solution of $D$. Then $\max D=\min P$ if and only if there exists $x^{*} \in$ $X\left(\lambda^{*}\right) \cap S$ such that

$$
\lambda_{i}^{*}\left[f_{i}\left(x^{*}\right)-b_{i}\right]=0, \quad i=1,2, \ldots, m .
$$




\section{Proof:}

The if part Let $\gamma^{*}$ be the optimal value of D. As $x^{*} \in X\left(\lambda^{*}\right), x^{*}$ is a global minimizer of $L\left(., \lambda^{*}\right)$ so that

$$
f_{0}\left(x^{*}\right)+\sum_{i=1}^{m} \lambda_{i}^{*}\left[f_{i}\left(x^{*}\right)-b_{i}\right]=\gamma^{*}=\max \mathrm{D}=f_{0}\left(x^{*}\right),
$$

and since $x^{*}$ is feasible, we have $\max \mathrm{D}=\min \mathrm{P}$, the desired result.

The only if part Let $x^{*}$ be a global minimizer of $\mathrm{P}$. If $\max \mathrm{D}=\min \mathrm{P}$, then we also have min $\mathrm{PP}=\min \mathrm{P}$ and the Dirac measure $\delta_{x^{*}}$ is solution of PP. Therefore, from Theorem 3(a) it follows that $\delta_{x^{*}}\left(X\left(\lambda^{*}\right)\right)=1$, that is, $x^{*} \in X\left(\lambda^{*}\right)$ (hence $x^{*} \in X\left(\lambda^{*}\right) \cap S$ ) and (15) follows from (13).

Corollary 4 is useful in global optimization. A "trial and error" method to find a global minimizer of $\mathrm{P}$ first computes a local minimizer $x^{*}$ of $\mathrm{P}$ with associated KKT multiplier $\lambda^{*}$, and then tries to check whether $x^{*}$ is a global minimizer, that is, if it is also a global minimizer of $L\left(., \lambda^{*}\right)$. On the contrary, Corollary 4 suggests to first solve $D$ (a convex problem) and then check whether there exists some $x^{*} \in X\left(\lambda^{*}\right) \cap S$ that satisfies (15). In some cases, the latter problem also reduces to a convex problem (see next Section on the general quadratic case). 
If we now denote by $\Delta$ the set of optimal solutions $\lambda$ of $\mathrm{D}$, we obtain as a corollary:

Corollary 5 Let Assumption A and condition B hold. If PP is solvable and

$$
\bigcap_{\lambda \in \Delta} X(\lambda)=\left\{x^{*}\right\}
$$

then $x^{*}$ is a global minimizer of $P$ and $\max D=\min P$.

Proof: From Theorem 3, let $\mu^{*}$ be an optimal solution of PP. Since, $\mu^{*}$ is concentrated on $X(\lambda)$ for every $\lambda \in \Delta$, from (16) we must have $\mu^{*}=\delta_{x^{*}}$, with $\delta_{x^{*}}$ the Dirac measure at $x^{*}$. But this implies that $x^{*}$ is feasible for $\mathrm{P}$ and $\min \mathrm{PP}=f\left(x^{*}\right) \leq \inf \mathrm{P}$, and thus, $\max \mathrm{D}=\min \mathrm{P}$.

\subsection{The (general) quadratic case}

Consider the case where the $f_{i}$ 's are quadratic functions, i.e.,

$$
x \mapsto f_{i}(x):=x^{\prime} Q_{i} x+2 c_{i}^{\prime} x, \quad i=0,1, \ldots, m,
$$

where $c^{\prime}$ denotes the transpose of a vector $c$. Denote also by $\langle A, B\rangle$ the usual scalar product trace $(\mathrm{AB})$ for real-valued symmetric matrices. 
Then D has the form

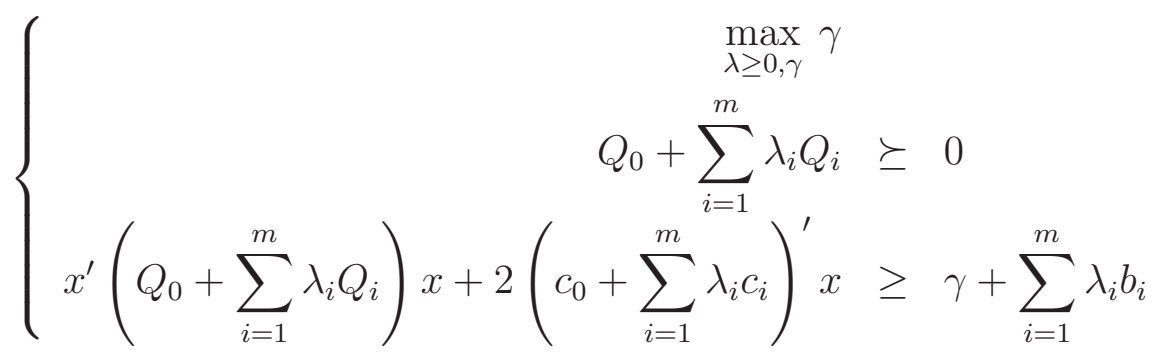

(the first constraint is necessary for $\sup \mathrm{D}>-\infty$ ) or, equivalently,

$$
\max _{\lambda \geq 0, \gamma}\left\{\gamma \mid\left[\begin{array}{cc}
Q_{0}+\sum_{i=1}^{m} \lambda_{i} Q_{i} & c_{0}+\sum_{i=1}^{m} \lambda_{i} c_{i} \\
\left(c_{0}+\sum_{i=1}^{m} \lambda_{i} c_{i}\right)^{\prime} & -\gamma-\sum_{i=1}^{m} \lambda_{i} b_{i}
\end{array}\right] \succeq 0\right\}
$$

an LMI problem whose dual $\mathrm{D}^{*}$

$$
\mathrm{D}^{*} \mapsto\left\{\begin{aligned}
\min & \left\langle Q_{0}, Y\right\rangle+2 c_{0}^{\prime} x \\
\text { s.t. } \quad\left\langle Q_{i}, Y\right\rangle+2 c_{i}^{\prime} x & \leq b_{i}, \quad i=1,2, \ldots, m \\
{\left[\begin{array}{cc}
Y & x \\
x^{\prime} & 1
\end{array}\right] } & \succeq 0
\end{aligned}\right.
$$

is a well-known relaxation of $\mathrm{P}$ (see e.g. Boyd and Vandenberghe [6] and the references therein). Under Assumption $\mathrm{A}$ and Condition $\mathrm{B}, \max \mathrm{D}=$ inf $\mathrm{PP}$. Therefore, if $\max \mathrm{D}=\min \mathrm{D}^{*}, \mathrm{PP}$ and $\mathrm{D}^{*}$ have same value. In fact, the equivalence of $\mathrm{D}^{*}$ and $\mathrm{PP}$ follows from the fact that to each positive 
semidefinite matrix $\left[\begin{array}{ll}1 & x^{\prime} \\ x & Y\end{array}\right]$, one may associate a probability measure $\mu$ with first moment vector $x$ and second-order moment matrix $Y$, that is,

$$
x_{i}=\int z_{i} d \mu, \quad \forall i=1, \ldots, n ; \quad Y(i, j)=\int z_{i} z_{j} d \mu, \quad \forall i, j=1, \ldots, n,
$$

for some probability $\mu$. Conversely, for every probability measure $\mu$, its vector $x$ of first-order moments and its matrix $Y$ of second-order moments must satisfy $\left[\begin{array}{ll}1 & x^{\prime} \\ x & Y\end{array}\right] \succeq 0$. Therefore, the above formulation $\mathrm{D}^{*}$ is just an equivalent formulation of PP since for every $i=0, \ldots, m, \int f_{i} d \mu$ only involves the first and second-order moments of $\mu$.

Let $\lambda^{*}$ be an optimal solution of $\mathrm{D}$ and let $\left\{u_{1}, \ldots, u_{p}\right\}$ be an orthonormal basis of $N:=\operatorname{Ker}\left(Q_{0}+\sum_{i=1}^{m} \lambda_{i}^{*} Q_{i}\right)$. Then, $x \in X\left(\lambda^{*}\right)$ if and only if $x=$ $z_{0}+\sum_{j=1}^{p} y_{j} u_{j}$, where $z_{0}$ solves

$$
\left[Q_{0}+\sum_{i=1}^{m} \lambda_{i}^{*} Q_{i}\right] z_{0}=-\left[c_{0}+\sum_{i=1}^{m} \lambda_{i}^{*} c_{i}\right] .
$$

Let $z_{0}$ be a particular solution of (17). Let $H_{i}$ be the real-valued $(p, p)$ symmetric matrix defined by $H_{i}(j k):=u_{j}^{\prime} Q_{i} u_{k}, 1 \leq j, k \leq p$, and let $d_{i}$ be the $p$-vector $d_{i}(j):=\left(c_{i}+2 Q_{i} z_{0}\right)^{\prime} u_{j}, 1 \leq j \leq p$. Then, invoking Corollary $4, \max \mathrm{D}=\min \mathrm{P}$ if and only if the following systems of quadratic equations/inequations in $R^{p}$ :

$$
\begin{aligned}
& y^{\prime} H_{i} y+d_{i}^{\prime} y=b_{i}-z_{0}^{\prime} Q_{i} z_{0}-c_{i}^{\prime} z_{0} \quad\left(\lambda_{i}^{*}>0\right) \\
& y^{\prime} H_{i} y+d_{i}^{\prime} y \leq b_{i}-z_{0}^{\prime} Q_{i} z_{0}-c_{i}^{\prime} z_{0} \quad\left(\lambda_{i}^{*}=0\right)
\end{aligned}
$$


has a solution.

Let $m=2, c_{i}=0, i=0,1,2$ (the pure quadratic case with 2 constraints) so that $z_{0}=0$, and let $\lambda^{*}$ be such that $\lambda_{i}^{*}>0$ for all $i=1,2$. As $d_{i}=0$ for all $j=1,2$, checking (18)-(19) is equivalent to checking whether the linear system

$$
\left\langle H_{i}, Z\right\rangle=b_{i}, \quad i=1,2,
$$

has a positive semidefinite solution $Z$, an LMI (convex) problem (see e.g. Corollary 20.3 in Barvinok [4]).

\section{The homogeneous case}

In this section we specialize the results to the case where the $f_{i}$ are all (positively) homogeneous polynomials with the same degree $p$, that is, for every scalar $\lambda>0$,

$$
f_{i}(\lambda x)=\lambda^{p} f_{i}(x), \quad \forall x \in X, i=0,1, \ldots, m .
$$

(In fact, it is also true for an arbitrary scalar $\lambda$.) A particular case of interest is when $p=2$, i.e., when the $f_{i}$ are all quadratic forms $x \mapsto f_{i}(x)=x^{\prime} Q_{i} x$ for some real-valued $(n, n)$-symmetric matrices $Q_{i}, i=0,1, \ldots, m$.

We first obtain a simplified expression of D, the solvability of PP (remember that the assumptions in Theorem 2 are not satisfied here) and show that there exist optimal solutions of PP with finite support. 
Theorem 6 Let Assumption A and Condition B hold. Then PP is solvable so that $\max D=\min P P$. The dual $D$ is:

$$
\max _{\lambda \geq 0}\left\{-\sum_{i=1}^{m} \lambda_{i} b_{i} \mid f_{0}(x)+\sum_{i=1}^{m} \lambda_{i} f_{i}(x) \geq 0, \quad x \in X\right\} .
$$

In addition, let $\lambda^{*}$ be an optimal solution of $D$. Then, there exists an optimal solution $\mu^{*}$ of PP which is a convex combination of at most $m+1$ Dirac measures at points $x_{j}$ that verify

$$
\nabla\left(f_{0}+\sum_{i=1}^{m} \lambda_{i}^{*} f_{i}\right)\left(x_{j}\right)=0, \quad j=1,2, \ldots, m+1 .
$$

The proof is relegated to Appendix D. We also obtain the following result that takes advantage of the fact that $X\left(\lambda^{*}\right)$ is a cone. Indeed, by homogeneity, the $\nabla f_{i}$ are all (positively) homogeneous polynomials of degree $p-1$, so that

$$
\nabla\left(f_{0}+\sum_{i=1}^{m} \lambda_{i}^{*} f\right)(x)=0 \Rightarrow \nabla\left(f_{0}+\sum_{i=1}^{m} \lambda_{i}^{*} f\right)(\alpha x)=0,
$$

for every scalar $\alpha>0$ (and in fact, every scalar $\alpha$ ).

Theorem 7 Assume that $f_{i}$ are all (positively) homogeneous polynomials with degree $p$, and let Assumption $A$ and Condition $B$ hold. Let $\lambda^{*}$ be an optimal solution to the convex problem $D$ such that $X\left(\lambda^{*}\right)$ is a one-dimensional 
cone, then

$$
\max D=\min P P=\min P .
$$

In addition, let $x_{0} \in X\left(\lambda^{*}\right)$, then $x^{*}:=\alpha x_{0}$ with $\alpha^{p}=b_{i} / f_{i}\left(x_{0}\right)\left(\right.$ with $\left.\lambda_{i}^{*}>0\right)$ is a global minimizer of $P$.

Proof: From Theorem 6, we already know that both D and PP are solvable so that $\max \mathrm{D}=\min \mathrm{PP}$. Let $x_{0}$ be an arbitrary solution in $X\left(\lambda^{*}\right)$. From (12) in Theorem 3, every solution $\mu^{*}$ of PP is a probability measure on $X\left(\lambda^{*}\right)$, that is, for every $f \in L_{1}(\mu)$,

$$
\int f(x) \mu(d x)=\int f\left(\alpha x_{0}\right) \nu(d \alpha)
$$

for some probability measure $\nu$ on the real line.

From the homogeneity of $f_{i}, i=0,1, \ldots, m$, we have

$$
\int f_{i} d \mu^{*}=f_{i}\left(x_{0}\right) \int \alpha^{p} d \nu \quad i=0,1, \ldots, m .
$$

Therefore, from (13)

$$
\int \alpha^{p} d \nu=\frac{b_{i}}{f_{i}\left(x_{0}\right)} \quad \text { whenever } \lambda_{i}^{*}>0 .
$$


In addition, as $\mu^{*}$ is admissible

$$
\begin{aligned}
b_{k} & \geq \int f_{k}(x) d \mu^{*} \\
& =f_{k}\left(x_{0}\right) \int \alpha^{p} d \nu, \quad \forall k=1,2, \ldots, m .
\end{aligned}
$$

But then the point $x^{*}:=\alpha_{0} x_{0}$ with

$$
\alpha_{0}^{p}=\frac{b_{i}}{f_{i}\left(x_{0}\right)} \text { for some } \lambda_{i}^{*}>0,
$$

satisfies

$$
f_{k}\left(x^{*}\right)=f_{k}\left(x_{0}\right) \alpha_{0}^{p}=\int f_{k}(x) d \mu^{*}, \quad \forall k=0,1, \ldots, m,
$$

which proves that $x^{*}$ is feasible and $f_{0}\left(x^{*}\right)=\min \mathrm{PP}$, so that we may conclude that $x^{*}$ is a global minimizer of $\mathrm{P}$.

In fact, Theorem 7 can be improved. In view of Corollary 5 , if $\Delta$ denotes the set of optimal solutions of $\mathrm{D}$, Theorem 7 is valid if only $\cap_{\lambda \in \Delta} X(\lambda)$ is one-dimensional.

\subsection{Example: Pure quadratic optimization}

Consider the following (pure) quadratic optimization problem

$$
\mathrm{P} \mapsto f^{*}=\min _{x}\left\{x^{\prime} Q_{0} x \mid x^{\prime} Q_{i} x \leq b_{i}, i=1,2, \ldots, m\right\}
$$


where all the $Q_{i}, i=0,1, \ldots, m$ are real-valued $(n, n)$-symmetric matrices. We make the following assumption:

\section{Assumption C:}

(i) $Q_{i}$ is positive semi-definite $i=1,2, \ldots, m$;

(ii) $Q_{0}$ has a unique negative eigenvalue (counting its multiplicity) and, whenever $x \neq 0, Q_{0} x=0 \Rightarrow Q_{i} x \neq 0$ for all $i=1,2, \ldots, m$;

(iii) There exist nonnegative coefficients $\lambda_{i}, i=1,2, \ldots, m$, such that $Q_{0}+$ $\sum_{i=1}^{m} \lambda_{i} Q_{i}$ is positive semi-definite;

(iv) Slater's condition holds, i.e., $x_{0}^{\prime} Q_{i} x_{0}<b_{i}, i=1,2, \ldots, m$ for some $x_{0}$.

From Theorem 6, the dual problem $\mathrm{D}$ associated with $\mathrm{P}$ reads:

$$
\mathrm{D} \mapsto \max _{\lambda \geq 0, \gamma}\left\{\gamma \mid x^{\prime} Q_{0} x+\sum_{i=1}^{m} \lambda_{i} x^{\prime} Q_{i} x \geq \gamma+\sum_{i=1}^{m} \lambda_{i} b_{i}, \quad \forall x \in X\right\} .
$$

or, equivalently,

$$
\mathrm{D} \mapsto \max _{\lambda \geq 0}\left\{-\sum_{i=1}^{m} \lambda_{i} b_{i} \mid Q_{0}+\sum_{i=1}^{m} \lambda_{i} Q_{i} \succeq 0\right\},
$$


(with " $A \succeq B$ " standing for $A-B$ positive semidefinite) which is called an "LMI" problem and can be solved efficiently via interior point methods (see e.g. [6] and the references therein).

Remark 8 This is a particular case of the general quadratic case considered in Section 2.4, and D has a dual $D^{*}$ which reads

$$
D^{*} \mapsto \min _{Y \in S^{n} ; Y \succeq 0}\left\{\left\langle Q_{0}, Y\right\rangle \mid\left\langle Q_{i}, Y\right\rangle \leq b_{i} \quad i=1,2, \ldots, m\right\}
$$

where $S^{n}$ denotes the space of real-valued $(n, n)$-symmetric matrices. $D^{*}$ is a well-known relaxation of $P$. The relationship between $D^{*}$ and $P P$ which are both duals of $D$ with same value, is as follows. Let $\mu^{*}$ be an optimal solution of $P P$, and let $Y \in R^{n \times n}$ be its matrix of second-order moments, that is,

$$
Y(i, j):=\int z_{i} z_{j} d \mu^{*} \quad \forall i, j=1, \ldots, n .
$$

Then, obviously, $Y \succeq 0$ and $D^{*}$ is an equivalent formulation of $P P$ for $\int f_{i} d \mu^{*}=\left\langle Q_{i}, Y\right\rangle$ for every $i=1, \ldots, n$. Conversely, every solution $Y \succeq 0$ of $D^{*}$ can be decomposed into a convex combination $\sum_{k} \alpha_{k} x_{k} x_{k}^{\prime}$ of rank-one matrices $x_{k} x_{k}^{\prime}$ (with $\sum_{k} \alpha_{k}=1$ ). The probability measure $\mu:=\sum_{k} \alpha_{k} \delta_{x_{k}}$ solves PP.

The following result is an application of Theorem 7 in the pure quadratic case.

Theorem 9 Under Assumption $C$, max $D=f^{*}=f_{0}\left(x^{*}\right)$ for every global optimal solution $x^{*}$ of $P$. 
Proof: One may check that C(iii)-(iv) imply that Assumption A and Condition B hold. Moreover, from C(i)-(ii), the null-space $N$ of $Q_{0}+\sum_{i=1}^{m} \lambda_{i}^{*} Q_{i}$ is at most one-dimensional. The conclusion follows from Theorem 7 .

Hence computing a global optimal solution for the non-convex problem $\mathrm{P}$ reduces to solving the convex "LMI" problem D. In fact, P is a "hidden" convex problem.

\section{References}

[1] E.J. Anderson And P. NAsh. Linear Programming in Infinite-Dimensional Spaces, John Wiley \& Sons, Chichester, 1987. E34, E39, E58

[2] A. Ben Tal and M. Teboulle. Hidden convexity in some nonconvex quadratically constrained quadratic programming, Math. Progr. 72, pp. 51-63, 1996. E30

[3] E.J. BALDer. Lectures on Young Measures, Cahiers de Mathématiques de la Décision, CEREMADE, Université Paris IX-Dauphine, 1994. E30, E34, E40

[4] A. Barvinok. Convexity, Duality, and Optimization, Lecture Notes, Department of Mathematics, The University of Michigan, 1998. E47, E64 
[5] P. Billingsley. Convergence of Probability Measures, Wiley, New York, 1968. E61

[6] L. Vandenberghe and S. Boyd. Semidefinite Programming, SIAM Review 38, pp. 49-95, 1996. E32, E45, E52

[7] B.D. Craven and J.J. Koliha, Genralizations of Farkas' theorem, SIAM J. Math. Anal. 8, pp. 983-997, 1977. E36

[8] J.B. Hiriart-Urruty. Optimisation et Analyse Convexe, PUF, Paris, 1998. E30

[9] J.B. Hiriart-Urruty. Conditions for global optimality 2, J. Global Optim., to appear. E30

[10] L.S. LAsdon, Optimization Theory for large Systems, The Macmillan Company, New York, 1970. E30, E37

[11] E. Mascolo and L. Migliacco, Relaxation methods in Control Theory, Appl. Math. Optim. 20, pp. 97-103, 1989. E30, E31, E34

[12] M.D. Perlman. Jensen's inequality for a convex vector-valued function on a infinite dimensional space, J. Multivariate Anal. 4, pp. 52-65, 1974. E38

[13] B.T. Polyak. Convexity of quadratic transformations and its use in control and optimization, J. Optim. Th. Appl. 99, pp. 553-583, 1998. E30 
[14] F. Topsoe. Topology and Measure, Lecture Notes in Mathematics, Springer, 1970. E64

[15] J. Warga, Optimal Control of Differential and Functional Equations, Academic Press, New York, 1972. E30, E31, E34

\section{A Proof of Theorem 1}

(i) We first prove the solvability of D. In view of Assumption A, P is of course feasible and therefore we have $\sup \mathrm{D}<+\infty$. In addition, since the feasible set of $\mathrm{D}$ is not empty, we also have sup $\mathrm{D}>-\infty$. Hence, consider a maximizing sequence $\left\{\gamma^{k}, \lambda_{i}^{k} \geq 0\right\}$, i.e., a sequence such that

$$
f_{0}(x)+\sum_{i=1}^{m} \lambda_{i}^{k}\left[f_{i}(x)-b_{i}\right] \geq \gamma^{k} \quad \forall x \in X .
$$

and

$$
\gamma^{k} \uparrow \sup \mathrm{D} \text {. }
$$

In particular, with $x_{j}$ as in Assumption $\mathrm{A}$,

$$
f_{0}\left(x_{j}\right)+\sum_{i=1}^{m} \lambda_{i}^{k}\left[f_{i}\left(x_{j}\right)-b_{i}\right] \geq \gamma^{k}
$$


which implies that $\lambda_{j}$ is bounded, otherwise

$$
f_{0}\left(x_{j}\right)+\sum_{i=1}^{m} \lambda_{i}^{k}\left[f_{i}\left(x_{j}\right)-b_{i}\right] \rightarrow-\infty,
$$

in contradiction with $\gamma_{k} \uparrow \sup$ D. Hence, with a similar argument, all the $\lambda_{i}$ are nonnegative and bounded above.

Therefore, there exist nonnegative scalars $\gamma^{*}, \lambda_{i}^{*}, i=1,2, \ldots, m$, and a subsequence $\left\{k_{j}\right\}$ such that, as $j \rightarrow \infty$,

$$
\gamma^{k_{j}} \rightarrow \gamma^{*} \text { and } \lambda_{i}^{k_{j}} \rightarrow \lambda_{i}^{*}, \quad i=1,2, \ldots, m
$$

so that, $\gamma^{*}=\sup \mathrm{D}$, and obviously (fix $x$ and let $j \rightarrow \infty$ in (27))

$$
f_{0}(x)+\sum_{i=1}^{m} \lambda_{i}^{*}\left[f_{i}(x)-b_{i}\right] \geq \gamma^{*} \quad \forall x \in X .
$$

Equivalently,

$$
\inf _{x}\left\{f_{0}(x)+\sum_{i=1}^{m} \lambda_{i}^{*}\left[f_{i}(x)-b_{i}\right]\right\}=\gamma^{*}=\sup \mathrm{D} .
$$

This proves that $\lambda^{*}$ solves $\mathrm{D}$, i.e., $\sup \mathrm{D}=\max \mathrm{D}$.

(ii) We now show that there is no duality gap between PP and D. Under Slater's condition, i.e., when there is some $x_{0} \in S$ such that $f_{i}\left(x_{0}\right)<b_{i}$ 
for all $i=1,2, \ldots, m$ the absence of a duality gap follows from e.g. Theorem 3.13, p. 55 in Anderson and Nash. Indeed, Slater's condition is just the interior point condition needed in that theorem. However, under the weaker Assumption A, such an interior point may not exist.

As $X:=R^{n}$ is locally compact separable, consider a nondecreasing sequence of compact sets $K_{i} \uparrow X, i=0,1, \ldots$, such that $K_{0}$ contains $x_{j}, j=1,2, \ldots, m$, with $x_{j}$ as in Assumption A. Let $D_{i}$ be the optimization problem

$$
\mathrm{D}_{i} \mapsto \sup _{\lambda \geq 0, \gamma}\left\{\gamma \mid f_{0}(x)+\sum_{k=1}^{m} \lambda_{k}\left[f_{k}(x)-b_{k}\right] \geq \gamma \quad \forall x \in K_{i}\right\},
$$

which is the dual of the optimization problem

$$
\mathrm{PP}_{i} \mapsto\left\{\begin{aligned}
\inf _{\mu \in \mathcal{M}\left(K_{i}\right), \mu \geq 0} \int f_{0} d \mu & \\
\int\left[f_{k}(x)-b_{k}\right] \mu(d x) & \leq 0, k=1,2, \ldots, m \\
\mu\left(K_{i}\right) & =1
\end{aligned}\right.
$$

In view of Assumption $\mathrm{A}$ and $\mathrm{B}$ and the fact that $K_{i}$ is compact, both $D_{i}$ and $P P_{i}$ are consistent with finite value. In addition, from the compactness of $K_{i}$, and the fact that the restrictions to $K_{i}$ of $f_{k}, k=$ $0,1, \ldots, m$ are continuous, there is no duality gap between $\mathrm{D}_{i}$ and $\mathrm{PP}_{i}$, and $\mathrm{PP}_{i}$ is solvable. Indeed, the feasible set of $\mathrm{PP}_{i}$ is a compact subset of $\mathcal{M}\left(K_{i}\right)$ for the weak* topology $\sigma\left(\mathcal{M}\left(K_{i}\right), C\left(K_{i}\right)\right.$ ) (with $C\left(K_{i}\right)$ the 
space of continuous functions on $K_{i}$ ), and $\int f_{0} d \mu$ is a continuous linear functional for that topology. Thus, $\mathrm{PP}_{i}$ is solvable. For the absence of a duality gap it suffices to prove that the set $H \subset R^{m+2}$, defined by

$$
H:=\left\{\left(T \mu+v,\langle 1, \mu\rangle, \int f_{0} d \mu\right) \mid v \in\left(R^{m}\right)^{+}, \mu \geq 0, \mu \in \mathcal{M}\left(K_{i}\right)\right\}
$$

is closed (see e.g. Theorem 3.9 in [1]), which also follows from compactness arguments.

In addition, with similar arguments as in (i), $\mathrm{D}_{i}$ is also solvable. Therefore, let $\mu_{i} \in \mathcal{M}\left(K_{i}\right)$ be an optimal solution of $\mathrm{PP}_{i}$ and let $\left\{\lambda^{i} \geq 0, \gamma^{i}\right\}$ be an optimal solution of $\mathrm{D}_{i}$. From the absence of a duality gap between $\mathrm{PP}_{i}$ and $\mathrm{DD}_{i}$, we have

$$
\inf \mathrm{PP}_{i}=\min \mathrm{PP}_{i}=\max \mathrm{D}_{i} \geq \sup \mathrm{D}=\max \mathrm{D} .
$$

Let $i \rightarrow \infty$ so that $\gamma^{i} \downarrow \gamma^{*} \geq \max$ D. It suffices to prove that $\gamma^{*}=$ $\max \mathrm{D}$ since then, the sequence $\mu_{i}$ which satisfies

$$
\int f_{0} d \mu_{i}=\gamma^{i} \downarrow \gamma^{*} \leq \inf \mathrm{PP}
$$

will be a minimizing sequence of PP with limit value $\gamma^{*}$.

Consider the sequence of $\left\{\lambda^{i}\right\}$. As $x_{j} \in K_{i}$ for all $i$, we have

$$
f_{0}\left(x_{j}\right)+\sum_{k=1}^{m} \lambda_{k}^{i}\left[f_{k}\left(x_{j}\right)-b_{k}\right] \geq \gamma^{i} \geq \gamma^{*}, \quad j=1,2, \ldots, m .
$$


Therefore, for each $k=1,2, \ldots, m$, the sequence $\left\{\lambda_{k}^{i}\right\}$ is bounded otherwise (32) yields a contradiction. Therefore, there is a subsequence $\left\{i_{p}\right\}$ and coefficients $\lambda_{k}^{*}, k=1,2, \ldots, m$ such that, as $p \rightarrow \infty$,

$$
\lambda_{k}^{i_{p}} \rightarrow \lambda_{k}^{*}, \quad k=1,2, \ldots, m .
$$

Now fix $x \in X$. As $K_{i} \uparrow X$, there is some $p_{0}$ such that $x \in K_{i_{p}}$ for every $p \geq p_{0}$, and therefore

$$
f_{0}(x)+\sum_{k=1}^{m} \lambda_{k}^{i_{p}}\left[f_{k}(x)-b_{k}\right] \geq \gamma^{i_{p}} .
$$

Letting $p \rightarrow \infty$ yields

$$
f_{0}(x)+\sum_{k=1}^{m} \lambda_{k}^{*}\left[f_{k}(x)-b_{k}\right] \geq \gamma^{*} .
$$

As $x$ was arbitrary, $\left(\lambda^{*}, \gamma^{*}\right)$ is feasible for D. This and $\gamma^{*} \geq \max \mathrm{D}$ proves that $\left(\lambda^{*}, \gamma^{*}\right)$ is an optimal solution of $\mathrm{D}$.

\section{B Proof of Theorem 2}

$\mathrm{PP}$ is consistent since $\mathrm{P}$ is. Therefore, consider a minimizing sequence $\left\{\mu_{n}\right\}$ in $\mathcal{M}(X)$, that is, from $\mu_{n}(X)=1$ and $\mu_{n} \geq 0$, a sequence in $\mathcal{P}(X)$ that 
satisfies

$$
\int f_{0} d \mu_{n} \downarrow \inf \mathrm{PP} ; \int f_{i} d \mu_{n} \leq b_{i}, \quad i=1,2, \ldots, m .
$$

As $f_{m}$ is inf-compact, there is some $M>0$ such that $x \mapsto w(x):=M+$ $f_{m}(x) \geq 1$ for all $x \in X$. Consider the associated subsequence of measures

$$
\varphi_{n}(B):=\int_{B} w d \mu_{n}, \quad B \in \mathcal{B}, n=1,2, \ldots
$$

The constraints $\int f_{i} d \mu_{n} \leq b_{i}, i=1,2, \ldots, m$ read

$$
\int \frac{f_{i}}{w} d \varphi_{n} \leq b_{i}, \quad i=1,2, \ldots, m
$$

and $\int f_{0} d \mu_{n} \downarrow \inf \mathrm{PP}$ reads $\int \frac{f_{0}}{w} d \varphi_{n} \downarrow \inf \mathrm{PP}$. As the $f_{i}$ are all continuous and $w \geq 1$, under Condition (b), the functions $f_{i} / w \in C_{0}(X), i=0,1, \ldots, m-1$, where $C_{0}(X)$ is the Banach space of continuous functions that vanish at infinity, equipped with the sup-norm, and whose topological dual is $\mathcal{M}(X)$.

From $\int w d \mu_{n}=\int f_{m} d \mu_{n}+M \leq b_{m}+M$, it follows that the sequence of measures $\left\{\varphi_{n}\right\}$ is norm-bounded, so that by weak* sequential compactness of the unit ball of $\mathcal{M}(X)$, there is a measure $\varphi$ and a subsequence $\left\{\varphi_{n_{k}}\right\}$ such that

$$
\lim _{k \rightarrow \infty} \int h d \varphi_{n_{k}}=\int h d \varphi, \quad \forall h \in C_{0}(X)
$$


and therefore, in particular, as $f_{i} / w \in C_{0}(X), \forall i \neq m$,

$$
\int \frac{f_{i}}{w} d \varphi=\lim _{k \rightarrow \infty} \int \frac{f_{i}}{w} d \varphi_{n_{k}} \leq b_{i}, \quad i=1,2, \ldots, m-1 .
$$

In addition,

$$
\int \frac{f_{0}}{w} d \varphi=\lim _{k \rightarrow \infty} \int \frac{f_{0}}{w} d \varphi_{n_{k}}=\inf \mathrm{PP} .
$$

Introduce the measure $\mu^{*}$ defined by $\mu^{*}(B):=\int_{B} w^{-1} d \varphi, B \in \mathcal{B}$, so that (33)(34) read

$$
\int f_{i} d \mu^{*} \leq b_{i}, \quad i=1,2, \ldots, m-1 ; \int f_{0} d \mu^{*}=\inf \mathrm{PP} .
$$

We next prove that $\mu^{*}$ is a probability measure. As $w$ is inf-compact, $w^{-1} \in$ $C_{0}(X)$, so that

$$
\begin{aligned}
\mu^{*}(X) & =\int w^{-1} d \varphi \\
& =\lim _{k \rightarrow \infty} \int w^{-1} d \varphi_{n_{k}} \\
& =\lim _{k \rightarrow \infty} \mu_{n_{k}}(X)=1 .
\end{aligned}
$$

Therefore, by the Portmanteau Theorem (see e.g. Billingsley [5]), the sequence $\varphi_{n_{k}}$ converges "weakly" to $\varphi$ and not only "weak", that is,

$$
\int h d \varphi_{n_{k}} \rightarrow \int h d \varphi, \quad \forall h \in C_{b}(X)
$$


It remains to prove that $\int f_{m} d \mu^{*} \leq b_{m}$. This follows from the definition of $w$. Indeed, as $f_{m} w^{-1} \in C_{b}(X)$, one has

$$
\begin{aligned}
\int f_{m} d \mu^{*}=\int f_{m} w^{-1} d \varphi & =\lim _{k \rightarrow \infty} \int f_{m} w^{-1} d \varphi_{n_{k}} \\
& =\lim _{k \rightarrow \infty} \int f_{m} d \mu_{n_{k}} \leq b_{m} .
\end{aligned}
$$

The latter combined with (35) implies that $\mu^{*}$ is admissible for PP and $\int f_{0} d \mu^{*}=\inf \mathrm{PP}$, which proves that $\mathrm{PP}$ is solvable.

\section{Proof of Theorem 3}

(a) Let $\left(\lambda^{*}, \gamma^{*}\right)$ be an optimal solution of $\mathrm{D}$ and let $\mu^{*}$ be an optimal solution of PP. From the absence of a duality gap between PP and D we have:

$$
\gamma^{*}=\int f_{0} d \mu^{*}
$$

In addition, from

$$
0 \leq f_{0}(x)+\sum_{i=1}^{m} \lambda_{i}^{*}\left[f_{i}(x)-b_{i}\right]-\gamma^{*}, \quad \forall x \in X,
$$


and $\int\left(f_{i}(x)-b_{i}\right) d \mu^{*} \leq 0$ for all $i=1,2, \ldots, m$, integration w.r.t. $\mu^{*}$ yields

$$
0=\sum_{i=1}^{m} \lambda_{i}^{*} \int\left(f_{i}(x)-b_{i}\right) d \mu^{*}
$$

Consequently, from the nonnegativity of each term, one has

$$
\lambda_{i}^{*} \int\left(f_{i} d \mu^{*}-b_{i}\right) d \mu^{*}=0, \quad i=1,2, \ldots, m,
$$

i.e. (13) holds and

$$
0=\int\left[f_{0}(x)+\sum_{i=1}^{m} \lambda_{i}^{*}\left[f_{i}(x)-b_{i}\right]-\gamma^{*}\right] d \mu^{*}
$$

This clearly implies that

$$
0=f_{0}(x)+\sum_{i=1}^{m} \lambda_{i}^{*}\left[f_{i}(x)-b_{i}\right]-\gamma^{*}, \quad \mu^{*} \text { a.e. }
$$

which yields (12), and (14) follows whenever the $f_{i}$ are all differentiable.

(b) Let $X\left(\lambda^{*}\right)$ be the compact set of global minimizers of $L\left(., \lambda^{*}\right)$ and let $\mu^{*}$ be an optimal solution of PP. We have just seen that $\mu^{*}$ is a probability measure on $X\left(\lambda^{*}\right)$. Thus, we could have replaced $X$ by $X\left(\lambda^{*}\right)$. The space $\mathcal{P}\left(X\left(\lambda^{*}\right)\right)$ of probability measures on $X\left(\lambda^{*}\right)$ is compact and 
convex for the weak* topology of $\mathcal{M}\left(X\left(\lambda^{*}\right)\right)$. By the Krein-Milman Theorem, $\mathcal{P}\left(X\left(\lambda^{*}\right)\right)$ is the weak* closure of the convex hull of its extreme points which are Dirac measures (see e.g. [14]).

Let $I^{*}$ be such that $i \in I^{*}$ if and only if $\int f_{i} d \mu^{*}=b_{i}$, i.e., $I^{*}$ denotes the set of active constraint at $\mu^{*}$.

The optimal value of PP is the same as if we had removed the constraints $i \notin I^{*}$ in $\mathrm{PP}$ and replaced the inequality by equality for $i \in I^{*}$. Indeed, if by removing the inactive constraints $i \notin I^{*}$, we obtain a strictly better solution $\nu$ (eventually with $\int f_{i} d \nu>b_{i}$ for some $i \notin I^{*}$ ) then there would be a convex combination $\mu:=\alpha \nu+(1-\alpha) \mu^{*}$ with $\alpha>0$, such that $\int f_{i} d \mu \leq b_{i}$ for all $i \notin I^{*}$ and with $\int f_{0} d \mu<\int f_{0} d \mu^{*}$, a contradiction.

Let $H_{i} \subset \mathcal{M}\left(X\left(\lambda^{*}\right)\right), i \in I^{*}$ be the hyperplanes $\left\{\mu \mid \int f_{i} d \mu=b_{i}\right\}$ associated with the active constraints at $\mu^{*}$. Since the $f_{i}$ are continuous, the $H_{i}$ are weak* closed and convex in $\mathcal{M}\left(X\left(\lambda^{*}\right)\right)$ so that $\mathcal{P}\left(X\left(\lambda^{*}\right)\right) \cap\left[\cap_{i \in I^{*}} H_{i}\right]$ is a convex set in $\mathcal{M}\left(X\left(\lambda^{*}\right)\right)$, compact for the weak $^{*}$ topology. In addition, the linear functional $\int f_{0} d \mu$ is continuous in that topology. Therefore, it attains its minimum at an extreme point which, by Caratheodory Theorem (see e.g. [4, Th. 28.2, p. 66]), is a convex combination of at most $s+1$ extreme points of $\mathcal{P}\left(X\left(\lambda^{*}\right)\right)$, i.e., $s+1$ Dirac measures on $X\left(\lambda^{*}\right)$. 


\section{Proof of Theorem 6}

From Theorem 1, D is solvable and there is no duality gap, that is max $\mathrm{D}=$ inf PP. Let $\left(\lambda^{*}, \gamma^{*}\right)$ be an optimal solution of $\mathrm{D}$ so that $\gamma^{*}:=\max \mathrm{D}$. As the $f_{i}$ 's are homogeneous polynomials, a minimizer $x^{*}$ of $f_{0}+\sum_{i} \lambda_{i}^{*}\left[f_{i}-b_{i}\right]$ satisfies

$$
\nabla\left(f_{0}+\sum_{i=1}^{m} \lambda_{i}^{*} f_{i}\right)\left(x^{*}\right)=0,
$$

and using the homogeneity of the $f_{i}$ 's (hence of the $\nabla f_{i}$ 's), we obtain

$$
\left(f_{0}+\sum_{i=1}^{m} \lambda_{i}^{*} f_{i}\right)\left(x^{*}\right)=0,
$$

which in turn yields,

$$
\gamma^{*}=-\sum_{i=1}^{m} \lambda_{i}^{*} b_{i}
$$

Hence, D simplifies to (20).

We now consider the solvability of PP. Let $K_{n} \uparrow X$ be the sequence of compact sets already considered in the proof of Theorem 1 . We already know that

$$
\max \mathrm{D}_{n}=\min \mathrm{PP}_{n} \downarrow \inf \mathrm{PP}=\max \mathrm{D}
$$


(cf. (31)), and

$$
\lambda^{n_{k}} \rightarrow \lambda^{*}, \quad \gamma_{n_{k}} \downarrow \gamma^{*}=\max \mathrm{D}
$$

for some subsequence $\left\{\lambda^{n_{k}}, \gamma_{n_{k}}\right\}$ of optimal solutions to $\mathrm{D}_{n}$. Now let $x_{n}$ be a minimizer of $\left(f_{0}+\sum_{i=1}^{m} \lambda_{i}^{n} f_{i}\right)$ on $K_{n}$. We must have

$$
\left(f_{0}+\sum_{i=1}^{m} \lambda_{i}^{n_{k}} f_{i}\right)\left(x_{n_{k}}\right)=\gamma_{n_{k}}+\sum_{i=1}^{m} \lambda_{i}^{n_{k}} b_{i} \geq \sum_{i=1}^{m}\left[\lambda_{i}^{n_{k}}-\lambda_{i}^{*}\right] b_{i},
$$

since $\gamma_{n_{k}} \downarrow \gamma^{*}$. In addition, given $\epsilon>0$, from the convergence of $\lambda^{n_{k}}$ to $\lambda^{*}$ it follows that

$$
\left(f_{0}+\sum_{i=1}^{m} \lambda_{i}^{n_{k}} f_{i}\right)\left(x_{n_{k}}\right) \geq-\epsilon
$$

for all $k$ sufficiently large, say $k \geq k_{0}$. Moreover, also from $\gamma_{n_{k}} \downarrow \gamma^{*}$, it follows that

$$
\left(f_{0}+\sum_{i=1}^{m} \lambda_{i}^{n_{k}} f_{i}\right)\left(x_{n_{k}}\right) \geq\left(f_{0}+\sum_{i=1}^{m} \lambda_{i}^{n_{k+p}} f_{i}\right)\left(x_{n_{k+p}}\right) \geq-\epsilon, \quad \forall p=1,2, \ldots
$$

and as $x_{n} \in K_{n+p}$ for all $p=1,2, \ldots$, it follows that

$\left(f_{0}+\sum_{i=1}^{m} \lambda_{i}^{n_{k+p}} f_{i}\right)\left(x_{n_{k}}\right) \geq\left(f_{0}+\sum_{i=1}^{m} \lambda_{i}^{n_{k+p}} f_{i}\right)\left(x_{n_{k+p}}\right) \geq-\epsilon, \quad \forall p=1,2, \ldots$ 
Now, fix $s \geq k_{0}$ and assume that

$$
\left(f_{0}+\sum_{i=1}^{m} \lambda_{i}^{n_{s}} f_{i}\right)\left(x_{n_{s}}\right)=-\delta<0 .
$$

Take $y=\alpha x_{n_{s}}$ with $\alpha>1$ sufficiently large to ensure $-\alpha^{p} \delta<-\epsilon$. From $K_{n} \uparrow X$, there is some $p_{0}$ such that $y \in K_{n_{s+p}}$ for all $p \geq p_{0}$ and thus,

$$
\left(f_{0}+\sum_{i=1}^{m} \lambda_{i}^{n_{s+p}} f_{i}\right)(y) \geq\left(f_{0}+\sum_{i=1}^{m} \lambda_{i}^{n_{s+p}} f_{i}\right)\left(x_{n_{s+p}}\right) \quad p=1,2, \ldots .
$$

Therefore,

$$
\begin{aligned}
\left(f_{0}+\sum_{i=1}^{m} \lambda_{i}^{n_{s+p}} f_{i}\right)(y) & =\alpha^{p}\left(f_{0}+\sum_{i=1}^{m} \lambda_{i}^{n_{s+p}} f_{i}\right)\left(x_{n_{s}}\right) \\
& =-\alpha^{p} \delta \quad[\text { by homogeneity }] \\
& \geq\left(f_{0}+\sum_{i=1}^{m} \lambda_{i}^{n_{s+p}} f_{i}\right)\left(x_{n_{s+p}}\right) \quad[\text { by (38)] } \\
& \geq-\epsilon \quad[\text { by }(36)],
\end{aligned}
$$

a contradiction.

Therefore, there is a subsequence $n_{k}$ such that for sufficiently large $k$,

$$
f_{0}(x)+\sum_{i=1}^{m} \lambda_{i}^{n_{k}} f_{i}(x) \geq 0 \quad \forall x \in K_{n_{k}}
$$


But, as $K_{i}$ has nonempty interior and $0 \in \operatorname{int} K_{i}$ for sufficiently large $i$, by homogeneity

$$
f_{0}(x)+\sum_{i=1}^{m} \lambda_{i}^{n_{k}} f_{i}(x) \geq 0, \quad \forall x \in X .
$$

But then $\gamma_{n_{k}}=-\sum_{i=1}^{m} \lambda_{i}^{n_{k}} b_{i}$ and $D_{n_{k}}$ is equivalent to solving

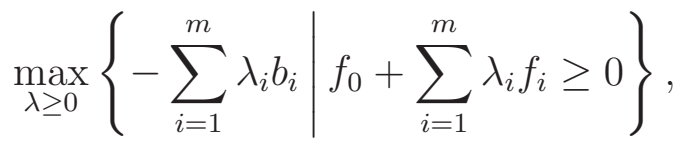

which is nothing less than solving D! Thus,

$$
\max \mathrm{D}_{n_{k}}=\gamma_{n_{k}}=\gamma^{*}=\max \mathrm{D}, \quad \forall k \geq k_{0} .
$$

As there is no duality gap between $\mathrm{D}_{n}$ and $\mathrm{PP}_{n}$ and both are solvable, from

$$
\inf \mathrm{PP}=\max \mathrm{D}=\max \mathrm{D}_{n}=\min \mathrm{PP}_{n},
$$

it follows that $\mathrm{PP}$ is solvable (take as $\mu^{*}$ an optimal solution of $\mathrm{PP}_{n_{k}}$ ).

Finally, as solving PP reduces to solving $\mathrm{PP}_{n}$ for some $n$ sufficiently large, an optimal solution of PP is a probability measure $\mu^{*}$ on a compact set $K_{n}$. That there is an optimal solution $\mu^{*}$ which is a convex combination of at most $m+1$ Dirac measures at points $x_{i}, i=1,2, \ldots, m+1$ then follows with similar arguments as in the proof of Theorem 3(b). That the $x_{i}$ 's satisfy (21) follows from the fact that $\mu^{*}$ must be concentrated on $X\left(\lambda^{*}\right)$ (cf. (14) in Theorem 3). 